\title{
Nanoscale
}

Check for updates

Cite this: Nanoscale, 2022, 14, 2167

Received 8th September 2021, Accepted 20th December 2021

DOI: $10.1039 / \mathrm{d} 1 \mathrm{nr} 05904 a$

rsc.li/nanoscale

\section{Ultra-clean high-mobility graphene on technologically relevant substrates $\uparrow$}

\author{
Ayush Tyagi, (iD a,b Vaidotas Mišeikis, iD *b,c Leonardo Martini, (iD ${ }^{b}$ Stiven Forti, (iD ${ }^{b}$ \\ Neeraj Mishra, b,c Zewdu M. Gebeyehu, iD b,c Marco A. Giambra, ' Jihene Zribi, \\ Mathieu Frégnaux, (D) e Damien Aureau, (D) e Marco Romagnoli, ${ }^{f}$ Fabio Beltram (iD) a \\ and Camilla Coletti (iD *b,c
}

Graphene grown via chemical vapour deposition (CVD) on copper foil has emerged as a high-quality, scalable material, that can be easily integrated on technologically relevant platforms to develop promising applications in the fields of optoelectronics and photonics. Most of these applications require low-contaminated highmobility graphene (i.e., approaching $10000 \mathrm{~cm}^{2} \mathrm{~V}^{-1} \mathrm{~s}^{-1}$ at room temperature) to reduce device losses and implement compact device design. To date, these mobility values are only obtained when suspending or encapsulating graphene. Here, we demonstrate a rapid, facile, and scalable cleaning process, that yields high-mobility graphene directly on the most common technologically relevant substrate: silicon dioxide on silicon $\left(\mathrm{SiO}_{2} / \mathrm{Si}\right)$. Atomic force microscopy (AFM) and spatially-resolved X-ray photoelectron spectroscopy (XPS) demonstrate that this approach is instrumental to rapidly eliminate most of the polymeric residues which remain on graphene after transfer and fabrication and that have adverse effects on its electrical properties. Raman measurements show a significant reduction of graphene doping and strain. Transport measurements of 50 Hall bars (HBs) yield hole mobility $\mu_{\mathrm{h}}$ up to $\sim 9000 \mathrm{~cm}^{2} \mathrm{~V}^{-1} \mathrm{~s}^{-1}$ and electron mobility $\mu_{\mathrm{e}}$ up to $\sim 8000 \mathrm{~cm}^{2} \mathrm{~V}^{-1}$ $\mathrm{s}^{-1}$, with average values $\mu_{\mathrm{h}} \sim 7500 \mathrm{~cm}^{2} \mathrm{~V}^{-1} \mathrm{~s}^{-1}$ and $\mu_{\mathrm{e}} \sim 6300 \mathrm{~cm}^{2}$ $\mathrm{V}^{-1} \mathrm{~s}^{-1}$. The carrier mobility of ultraclean graphene reaches values nearly double than those measured in graphene processed with acetone cleaning, which is the method widely adopted in the field. Notably, these mobility values are obtained over large-scale and without encapsulation, thus paving the way to the adoption of graphene in optoelectronics and photonics.

\footnotetext{
${ }^{a}$ NEST, Scuola Normale Superiore, Piazza San Silvestro 12, 56127 Pisa, Italy ${ }^{b}$ Center for Nanotechnology Innovation @NEST, Istituto Italiano di Technologia, Piazza San Silvestro 12, 56127 Pisa, Italy.E-mail: vaidotas.miseikis@iit.it, camilla.coletti@iit.it

${ }^{c}$ Graphene Labs, Istituto Italiano di Tecnologia, via Morego 30, 16163 Genova, Italy

${ }^{d}$ CamGraPhIC srl, 56124 Pisa, Italy

${ }^{e}$ Institut Lavoisier de Versailles UMR 8180 Université Paris-Saclay, UVSQ, CNRS, 78035 Versailles, France

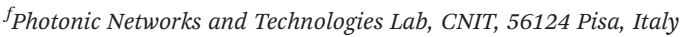

$\dagger$ Electronic supplementary information (ESI) available. See DOI: 10.1039/ d1nr05904a
}

\section{Introduction}

In the last years, graphene has shown its potential in numerous technological applications because of its many useful properties such as high electrical and thermal conductivity. ${ }^{1,2}$ In particular, thanks to tremendous progress made in the field of scalable graphene synthesis via chemical vapour deposition (CVD), wafer-scale graphene is now accessible and ready to be integrated for different applications in fields ranging from photonics, to optoelectronics, to sensing. ${ }^{3-8}$ Most of these applications require high-mobility ultra-clean graphene directly on a technologically-relevant substrate such as silicon dioxide on silicon $\left(\mathrm{SiO}_{2} / \mathrm{Si}\right){ }^{59}$ In particular, photonic devices with performance that is competitive with that of conventional technology require graphene with charge-carrier mobility near $10000 \mathrm{~cm}^{2} \mathrm{~V}^{-1} \mathrm{~s}^{-1}$ at carrier density $\sim 10^{12} \mathrm{~cm}^{-2}$ (ref. 9) in order to improve Seebeck coefficient in photothermal effect detectors ${ }^{10}$ and extinction ratio in photonic electro-absorption modulators. ${ }^{9-11}$ High mobility is also desirable to limit propagation losses and allow for reduced geometrical footprint. ${ }^{9}$ Also, low contamination is a requirement of foundries in which CVD graphene is included in integration process flows. The contaminant threshold for back-end-of-line in a CMOS fab is $10^{12}$ atoms per $\mathrm{cm}^{2}$ whereas in the front-end-of-line the threshold is two orders of magnitude more stringent. ${ }^{12,13}$

Since state-of-the-art (SOTA) scalable graphene is presently obtained via chemical vapour deposition (CVD) on metal substrates, ${ }^{8}$ the standard fabrication of graphene devices requires: (i) an unavoidable transfer step involving coating the graphene with polymeric resist (which acts as the support layer during the transfer) and (ii) optical or e-beam lithography (EBL). Polymethyl methacrylate (PMMA), in particular, is widely used for fabrication as well as transfer ${ }^{14}$ of CVD graphene. A wellknown issue in graphene processing is the presence of PMMA residues on graphene due to strong physical and chemical adsorption effects. ${ }^{15}$ Owing to the monolayer nature of graphene, surface adsorbates can induce carrier scattering, thus reducing the resulting mobility. ${ }^{16}$ To realize high-performing 
opto-electronic and photonic devices of technological relevance, flat and contaminant-free graphene over large scale is essential. Various methods have been used to address the issue of the polymer contamination on graphene, including stencil mask lithography, ${ }^{17}$ mechanical cleaning with the tip of an atomic force microscope (AFM) ${ }^{18,19}$ current-induced cleaning, ${ }^{20}$ PMMA degradation by laser treatment, ${ }^{21,22}$ hightemperature annealing ${ }^{23-25}$ and wet chemical cleaning, ${ }^{23,26,27}$ though each of these presents its own drawbacks. Stencil mask lithography relies on a physical mask placed in close proximity to the sample to define the metallic contacts or an etching pattern in graphene. While this does not require subjecting graphene to any polymer, the fragility of the masks imposes a compromise between the size of the patterning area and resolution. Furthermore, it does not allow the flexibility offered by EBL for rapid device prototyping. An effective cleaning of polymer residues from the graphene surface was demonstrated by "sweeping" it with an AFM tip operated in contact mode, however, this method is constrained to clean local areas only (typically, on the order of tens of microns) and is very time-consuming. Similar constraints apply to current- and laser-induced cleaning. Thermal annealing is compatible with large-scale processing, but, when performed on graphene/ $\mathrm{Si}-\mathrm{SiO}_{2}$, it was shown to increase doping and decrease mobility by inducing strong interactions between graphene and the substrate. $^{24,28}$ To date, wet chemical cleaning is the most adopted approach to prepare graphene prior to device implementation. ${ }^{23}$ When measuring large-area samples, (i.e. chips containing more than 10 test structures) in ambient conditions, carrier mobility typically does not exceed $5000 \mathrm{~cm}^{2}$ $\mathrm{V}^{-1} \mathrm{~s}^{-1}{ }^{8,29}$ We note that higher mobility values can be achieved for samples measured in vacuum ${ }^{26,30,31}$ or using modified growth strategies such as using polystyrene as growth precursor $^{32}$ or using stacks of $\mathrm{Cu}$ foam for super-clean growth. ${ }^{30}$ In the latter case, carrier mobility as high as $18500 \mathrm{~cm}^{2} \mathrm{~V}^{-1} \mathrm{~s}^{-1}$ has been reported. ${ }^{30}$ Chemical cleaning of polymer residues from graphene is sometimes done using strong solvents such as $N$-methyl-2-pyrrolidone (NMP), but they can induce lattice defects or delamination of graphene from the substrate. ${ }^{33}$

In this work, we demonstrate that by using a two-step wet chemical process after graphene transfer and device fabrication, graphene surface cleanliness and electrical performance are significantly improved with respect to other chemical treatments used so far. ${ }^{14,23}$ We perform a systematic comparison of CVD-grown graphene processed with standard single-step cleaning (1SC) in acetone and two-step cleaning (2SC) in acetone and remover AR 600-71, the latter being a two-component solvent. We also analyse our samples after the full fabrication cycle $(\mathrm{FF})$ using $2 \mathrm{SC}$ after each processing step (i.e. graphene transfer, etching and metal contact deposition). We use AFM, Raman spectroscopy, X-ray photoelectron spectroscopy (XPS) and charge-carrier transport measurements to highlight the improvement in morphological and electrical transport properties in graphene processed with 2SC. While AFM and XPS measurements confirm the effectiveness of 2SC in removing PMMA residues, Raman spectroscopy indicates reduction of doping and strain inhomogeneity. Electrical transport measurements performed on a chip containing 50 graphene HBs fabricated using 2SC show average hole mobility $\mu_{\mathrm{h}} \sim 7500 \mathrm{~m}^{2} \mathrm{~V}^{-1} \mathrm{~s}^{-1}$ and average electron mobility $\mu_{\mathrm{e}} \sim 6300 \mathrm{~m}^{2} \mathrm{~V}^{-1} \mathrm{~s}^{-1}$, i.e. an improvement of $65 \%$ and $37 \%$, respectively, compared to a sample processed with 1SC. The improved carrier mobility values are verified over several chips fabricated using the new cleaning process.

\section{Experiment and methods}

Single-crystal graphene arrays $^{34,35}$ with a lateral size of 200-250 $\mu \mathrm{m}$ were synthesized via CVD on $2 \times 2 \mathrm{~cm}^{2}$ electropolished Cu-foils ( $25 \mu \mathrm{m}$ thick, Alfa Aesar, purity $99.8 \%$ ) by following the procedure reported by Miseikis et al. ${ }^{36}$ Specifically, graphene was synthesized at a temperature of $1060{ }^{\circ} \mathrm{C}$ in a coldwall CVD reactor (Aixtron BM Pro) under argon (Ar), hydrogen $\left(\mathrm{H}_{2}\right)$ and methane $\left(\mathrm{CH}_{4}\right)$ with flow ratio of $900: 100: 1$, respectively. Afterwards, the graphene crystals were transferred on highly-doped $\mathrm{Si}$ substrates with a $285 \mathrm{~nm}$ layer of $\mathrm{SiO}_{2}$ (Siltronix) using a semi-dry technique as reported previously. ${ }^{36,37}$ A poly(methyl methacrylate) (PMMA) layer was used to support the graphene single-crystals while detaching them from Cu-foil via electrochemical delamination. ${ }^{38,39}$ The PMMA-coated graphene single crystals were then finally deposited on the target $\mathrm{SiO}_{2} / \mathrm{Si}$ substrate using a micrometric mechanical stage. More details about the graphene-transfer technique can be found in the ESI. $\dagger$ After transferring graphene from $\mathrm{Cu}$ to $\mathrm{SiO}_{2} / \mathrm{Si}$, a wet chemical cleaning method was used to remove the PMMA layer. For 1SC, the graphene sample was immersed in acetone for 2 hours and rinsed in isopropyl alcohol for 5 minutes, then dried under compressed nitrogen flow. In the case of $2 \mathrm{SC}$, the steps of $1 \mathrm{SC}$ were followed by a $3 \mathrm{~min}$ bath in remover AR 600-71 and a $10 \mathrm{~s}$ rinse in deionized water, followed by drying with compressed nitrogen. AR 600-71 (Allresist) is a two-component solvent (70\%, 1,3-dioxolane and 30\%, 1-methoxy 2-propanol), effective at stripping PMMA, Chemical Semi Amplified Resist (CSAR) and novolac-based resists. ${ }^{40}$ The two-step cleaning procedure was used after graphene transfer as well as after each fabrication step where PMMA removal was involved, i.e. after graphene etching and metal lift-off. During preliminary tests, 1,3-dioxolane and 1-methoxy 2-propanol (Sigma-Aldrich) were also used separately to assess the efficacy of the solvent constituents.

To elucidate the morphology of the graphene surface, AFM was performed with a Dimension ICON-PT (Bruker). Topographic images were obtained in peak force tapping mode (Bruker Scan-Asyst). ${ }^{41}$ Gwyddion software was used to process the AFM images, extract the surface profile and to perform surface roughness calculations and particle analysis.

Raman spectroscopy was performed with a Renishaw InVia system with a $532 \mathrm{~nm}$ laser, and a 100× objective, giving a spot size of $\sim 0.8 \mu \mathrm{m} .{ }^{8}$ Laser power was set to $\sim 1 \mathrm{~mW}$ to minimize 
heating. Raman mapping was performed using a motorised stage, over an area of $12 \times 12 \mu \mathrm{m}^{2}$ with a step size of $1 \mu \mathrm{m}$.

XPS analyses of the transferred graphene crystal were conducted on a Thermo Fisher Scientific Escalab 250 xi, equipped with a monochromatic Al-K $\alpha$ anode (1486.6 eV). Two complementary approaches were used to characterize the samples before and after the 1SC and 2SC process: parallel XPS Imaging for flakes localization and selected Small-area XPS (SAXPS) for spectroscopy. Parallel XPS Imaging is an acquisition mode using a large X-ray spot (i.e. $900 \mu \mathrm{m}$ ). Photoelectrons from the entire defined field of view (250 or $500 \mu \mathrm{m})$ are simultaneously collected on the 2D detector. Electrons of a given kinetic energy are focused on the channelplate detector to produce a direct image of the sample without scanning. By integrating images from consecutive energies, an average spectrum of the considered area can be generated. Maps were recorded in the energy range of $\mathrm{C} 1 \mathrm{~s}, \mathrm{O} 1 \mathrm{~s}$ and $\mathrm{Si}$ 2 p, using a $200 \mathrm{eV}$ pass energy and $0.1 \mathrm{eV}$ energy step between each acquisition. SAXPS was performed on the flakes evidenced by the previous map. This method maximizes the detected signal coming from a specific area (60 $\mu \mathrm{m}$ here) and minimizes the signal from the surrounding area. It is achieved by using irises and the spectrometer's transfer lens to flood the area with X-rays but limit the area from which the photoelectrons are collected. High-energy resolution spectral windows of interest were recorded for $\mathrm{C} 1 \mathrm{~s}, \mathrm{O}$ 1s and $\mathrm{Si} 2 \mathrm{p}$ core levels. The photoelectron detection was performed using a constant analyser energy (CAE) mode (10 eV pass energy) and a $0.05 \mathrm{eV}$ energy step. All the associated binding energies were corrected with respect to the $\mathrm{C} 1 \mathrm{~s}$ at $284.5 \mathrm{eV}$.

Electrical transport properties of CVD graphene were investigated by fabricating several chips with multiple Hall bars using EBL. One chip with 28 HBs was processed using 1SC. 2SC was used to fabricate a chip with $50 \mathrm{HBs}$, along with 3 other samples containing fewer (5-8) HBs to verify the results. Lithography was performed using a Zeiss UltraPlus scanning electron microscope (SEM) at $20 \mathrm{kV}$ and Raith Elphy Multibeam EBL system. The patterns were defined in positive e-beam resist (PMMA 950k, Allresist GMBH). Graphene was etched using oxygen plasma in a parallel-plate reactive ion etching (RIE) system (Sistec) at $35 \mathrm{~W}$ with $\mathrm{Ar} / \mathrm{O}_{2}$ flow of 5/80 sccm, respectively. The contacts were deposited by thermal evaporation of $60 \mathrm{~nm}$ of $\mathrm{Au}$ with a $7 \mathrm{~nm}$ Ni adhesion layer. Electrical transport characterisation was performed in ambient conditions using a custom-made probe station with tungsten tips on micropositioners. Electric field effect was measured using a pair of Keithley 2450 source-measure units, for a 4-terminal resistance measurement and back-gate sweep.

\section{Results and discussion}

\subsection{AFM characterization}

To evaluate the effectiveness of our cleaning procedure, the surface morphology of graphene after 1SC and 2SC were studied via AFM. A $10 \times 10 \mu \mathrm{m}^{2}$ area was selected near the edge of a graphene crystal to allow the analysis of polymer residues on graphene and $\mathrm{SiO}_{2}$ surface. The topographical images of the selected area are shown in Fig. 1a (1SC) and Fig. 1b (2SC). The $1 \mathrm{~S}$ cleaning protocol leaves nanometre-sized particles on graphene. ${ }^{42}$ When subjected to the second cleaning step, a remarkable reduction of polymer residues can be observed, revealing a flat and homogeneous graphene surface with only occasional wrinkles. Fig. 1c shows two-line profiles extracted from the same area after 1SC (blue curve) and 2SC (red curve). The former is dominated by surface height variation of $0-3 \mathrm{~nm}$, with a number of surface features reaching the height of almost $10 \mathrm{~nm}$. In the case of 2SC, the surface height variation is much less pronounced, with only a few points reaching a height of $\sim 2 \mathrm{~nm}$, corresponding mostly to the surface height variation of the $\mathrm{SiO}_{2}$ substrate, as can be seen outside of the graphene crystal. RMS roughness of the surface was measured on the graphene-coated area to be $\sim 2.8 \mathrm{~nm}$ after $1 \mathrm{SC}$ and reduced to $\sim 0.6 \mathrm{~nm}$ after $2 \mathrm{SC}$, nearly matching the intrinsic roughness of the $\mathrm{Si} / \mathrm{SiO}_{2}$ wafer $(\sim 0.5 \mathrm{~nm}$, declared by the supplier and verified by an AFM measurement before graphene deposition).

Particle analysis was done on a $10 \times 10 \mu \mathrm{m}^{2}$ area from the centre of a graphene crystal not including bare $\mathrm{SiO}_{2}$ (shown in Fig. S2a and b in ESI $\dagger$ ). For 1SC, 810 particles were counted, with an average height of $\sim 14 \pm 5 \mathrm{~nm}$ and an average radius of $\sim 19 \pm 13 \mathrm{~nm}$. After the $2^{\text {nd }}$ cleaning step, the number of particles was reduced to 34 , with an average height of $\sim 13 \pm 3 \mathrm{~nm}$ and an average radius of $\sim 20 \pm 14 \mathrm{~nm}$. Statistical distribution of particle height and radius of the sample after each cleaning step is shown in Fig. 1d and e, respectively. These results indicate that $>95 \%$ of surface contaminants are removed by $2 \mathrm{SC}$ compared to standard acetone cleaning. We also note that similar results were obtained on polycrystalline graphene wet transferred onto $\mathrm{Si} / \mathrm{SiO}_{2}$ (Fig. S6 $\mathrm{S}^{\dagger}$ ). The RMS roughness values obtained from $3 \times 3 \mu^{2}$ surface of wet-transferred graphene were $2.2 \mathrm{~nm}$ after $1 \mathrm{SC}$ and $0.7 \mathrm{~nm}$ after 2SC. Preliminary experiments were performed to understand whether one of the two constituents of remover AR 600-71, i.e., 1,3-dioxolane and 1-methoxy-2-propanol, had a major influence on removing particles: we found that the latter is the component yielding cleaner surfaces at AFM analysis, although less effective than remover AR 600-71, indicating that a synergic effect of the two is needed.

\subsection{Raman analysis}

Raman spectroscopy was performed on the sample to estimate graphene quality including doping and strain, after each processing and cleaning step. Fig. 2a shows representative Raman spectra obtained after graphene transfer and 1SC (black), 2SC (orange) and FF (green). Full fabrication corresponds to three rounds of PMMA deposition and 2SC. After 1SC, the spectrum of graphene shows the characteristic Raman G and 2D peaks with positions $\operatorname{Pos}(\mathrm{G}) \sim 1586.5 \pm 0.8 \mathrm{~cm}^{-1}$ and $\operatorname{Pos}(2 \mathrm{D}) \sim 2679$ $\pm 1.4 \mathrm{~cm}^{-1}$, respectively, with an average full width at halfmaximum (FWHM) of the G peak $\sim 15 \pm 1.2 \mathrm{~cm}^{-1}$ and average FWHM(2D) of $\sim 30.5 \pm 1.0 \mathrm{~cm}^{-1}$, which can be fitted with a 
a

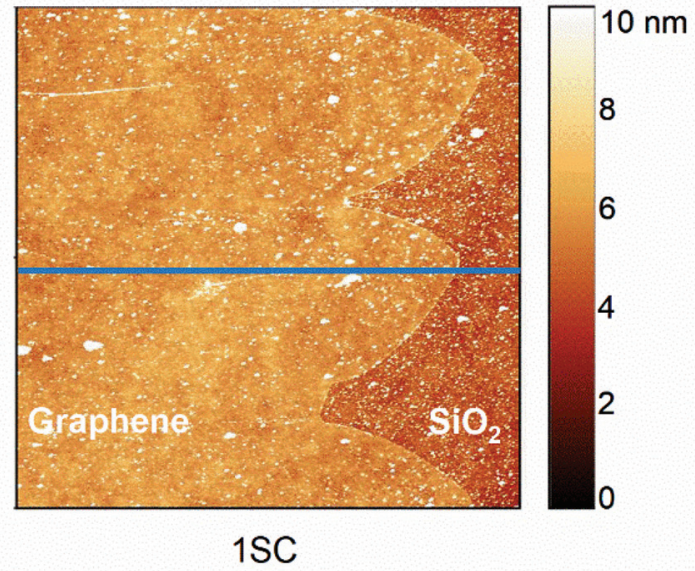

b

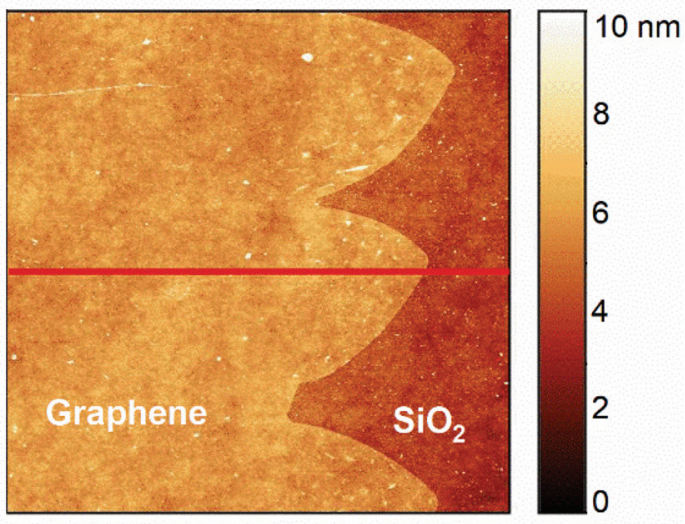

2SC
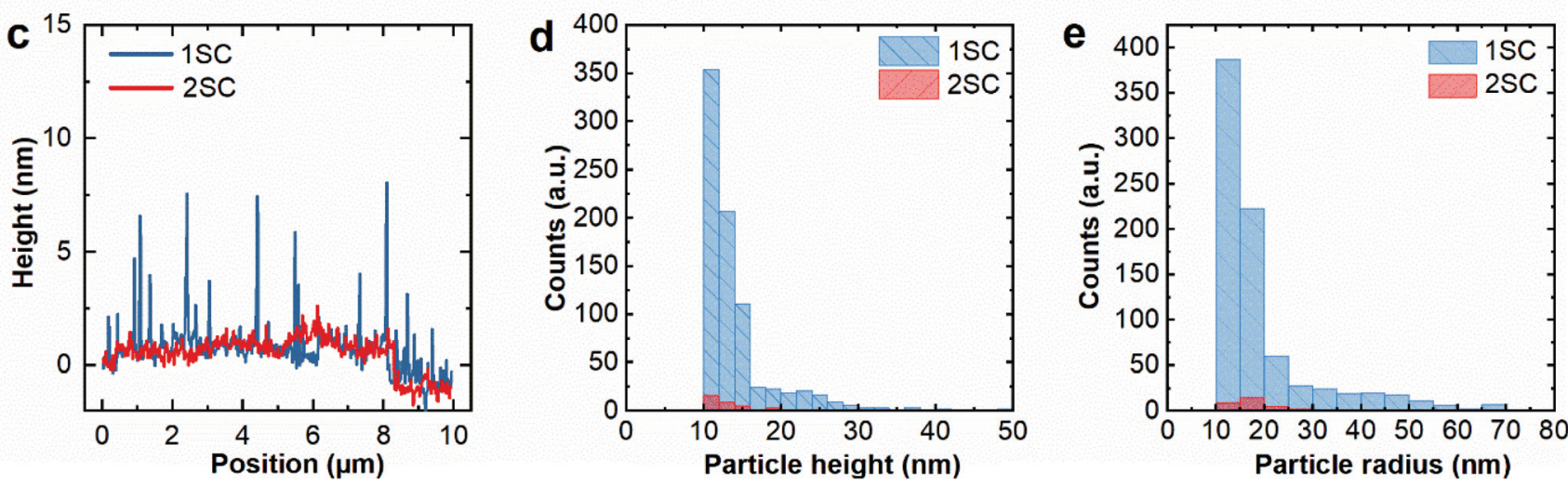

Fig. 1 (a) Topography image $\left(10 \times 10 \mu \mathrm{m}^{2}\right)$ of graphene crystal edge after transfer to $\mathrm{SiO}_{2} / \mathrm{Si}$ and $1 \mathrm{SC}$. (b) The same area after the second cleaning step. (c) Surface profile of graphene taken from the topographical images after 1SC (blue) and 2SC (red). (d) and (e) Statistical distribution of particle height and radius, respectively, after single- and two-step cleaning.

single Lorentzian, as expected for single-layer graphene. ${ }^{43}$ The D peak near $1350 \mathrm{~cm}^{-1}$ is absent, indicating a negligible amount of defects. ${ }^{44}$ Statistical Raman data obtained from a $12 \times 12 \mu \mathrm{m}^{2}$ map is shown in Fig. $2 \mathrm{~b}-\mathrm{e}$.

As shown in Fig. $2 \mathrm{~b}$, the $2 \mathrm{D}$ and $\mathrm{G}$ peak intensity ratio increases from $I(2 \mathrm{D}) / I(\mathrm{G}) \sim 2.8 \pm 0.2$ after transfer to $I(2 \mathrm{D}) / I(\mathrm{G})$ $\sim 3.1 \pm 0.5$ after $2 \mathrm{SC}$ and reaching $\sim 4.6 \pm 0.7$ after FF. The peak area ratio after transfer is $A(2 \mathrm{D}) / A(\mathrm{G}) \sim 5.7 \pm 0.4$, increasing to $A(2 \mathrm{D}) / A(\mathrm{G}) \sim 6 \pm 0.4$ after $2 \mathrm{SC}$ and $A(2 \mathrm{D}) / A(\mathrm{G}) \sim 7.9 \pm 0.6$ after FF. We use the $2 \mathrm{D} / \mathrm{G}$ peak area ratio, Fig. 2c, to estimate the doping in our samples at various stages of processing, by adapting the methodology reported by Basko et al. ${ }^{45}$ (more details provided in ESI $\dagger$ ). After transfer and 1SC, the doping is estimated to be $\sim(2.6 \pm 0.5) \times 10^{12} \mathrm{~cm}^{-2}$, reducing to $\sim(2.2 \pm$ $0.4) \times 10^{12} \mathrm{~cm}^{-2}$ after $2 \mathrm{SC}$ process. After $\mathrm{FF}$, it is further reduced to $\sim(1.0 \pm 0.3) \times 10^{12} \mathrm{~cm}^{-2}$.

We note that $\operatorname{Pos}(\mathrm{G})$ and $\operatorname{Pos}(2 \mathrm{D})$ in graphene are affected not only by doping ${ }^{46}$ but also by strain. ${ }^{47}$ The rates of change of the two peak positions as a function of strain are determined by the Grüneisen parameters, ${ }^{48}$ and the relative peak shift is typically observed ${ }^{47,49,50}$ to be $\Delta \operatorname{Pos}(2 \mathrm{D}) / \Delta \operatorname{Pos}(\mathrm{G}) \sim 2-3$.
As indicated by the solid lines in Fig. 2d, in our samples, the relative slope of $\Delta \operatorname{Pos}(2 \mathrm{D}) / \Delta \operatorname{Pos}(\mathrm{G})$ is $\sim 1.45$ after $1 \mathrm{SC}, \sim 1.06$ after $2 \mathrm{SC}$ and $\sim 1.25$ after $\mathrm{FF}$, respectively, indicating an inhomogeneous distribution of doping and strain. Uniaxial strain presents G-peak splitting, ${ }^{47}$ which can help to distinguish it from biaxial strain, ${ }^{51}$ though for small strain $(\lesssim 0.5 \%)$ the splitting cannot be resolved ${ }^{47,49}$ and we cannot rule out the presence or coexistence of both uniaxial and biaxial strain in our samples.

The shift of $\operatorname{Pos}(\mathrm{G})$ can be used to estimate strain, considering $\Delta \operatorname{Pos}(\mathrm{G}) / \Delta \varepsilon \sim 23(60) \mathrm{cm}^{-1} \%^{-1}$ for uniaxial (biaxial) strain..$^{47,49}$ In our samples, the average Pos(G) was measured at $\sim 1586.5 \pm 0.8 \mathrm{~cm}^{-1}$ after 1SC, $\sim 1583.5 \pm 1.2 \mathrm{~cm}^{-1}$ after $2 \mathrm{SC}$ and $\sim 1581.9 \pm 0.6 \mathrm{~cm}^{-1}$ after full fabrication, respectively. Considering that unstrained, intrinsic graphene $e^{43,52}$ is expected to have $\operatorname{Pos}(\mathrm{G}) \sim 1581.5 \mathrm{~cm}^{-1}$, and accounting for the effect of doping ${ }^{46,50}$ which we calculate from $A(2 \mathrm{D}) / A(\mathrm{G})$ (see above), we estimate the contribution of strain to $\Delta \operatorname{Pos}(\mathrm{G})$ to be $\sim 1.9 \pm 0.9 \mathrm{~cm}^{-1}$ after 1SC, $\sim 0.2 \pm 0.4 \mathrm{~cm}^{-1}$ after $2 \mathrm{SC}$ and $\sim 0.3 \pm 0.2 \mathrm{~cm}^{-2}$ after FF. This corresponds to uniaxial (biaxial) strain of $0.04 \%(0.02 \%)$ to $0.13 \%(0.05 \%)$ after $1 \mathrm{SC},-0.01 \%$ 

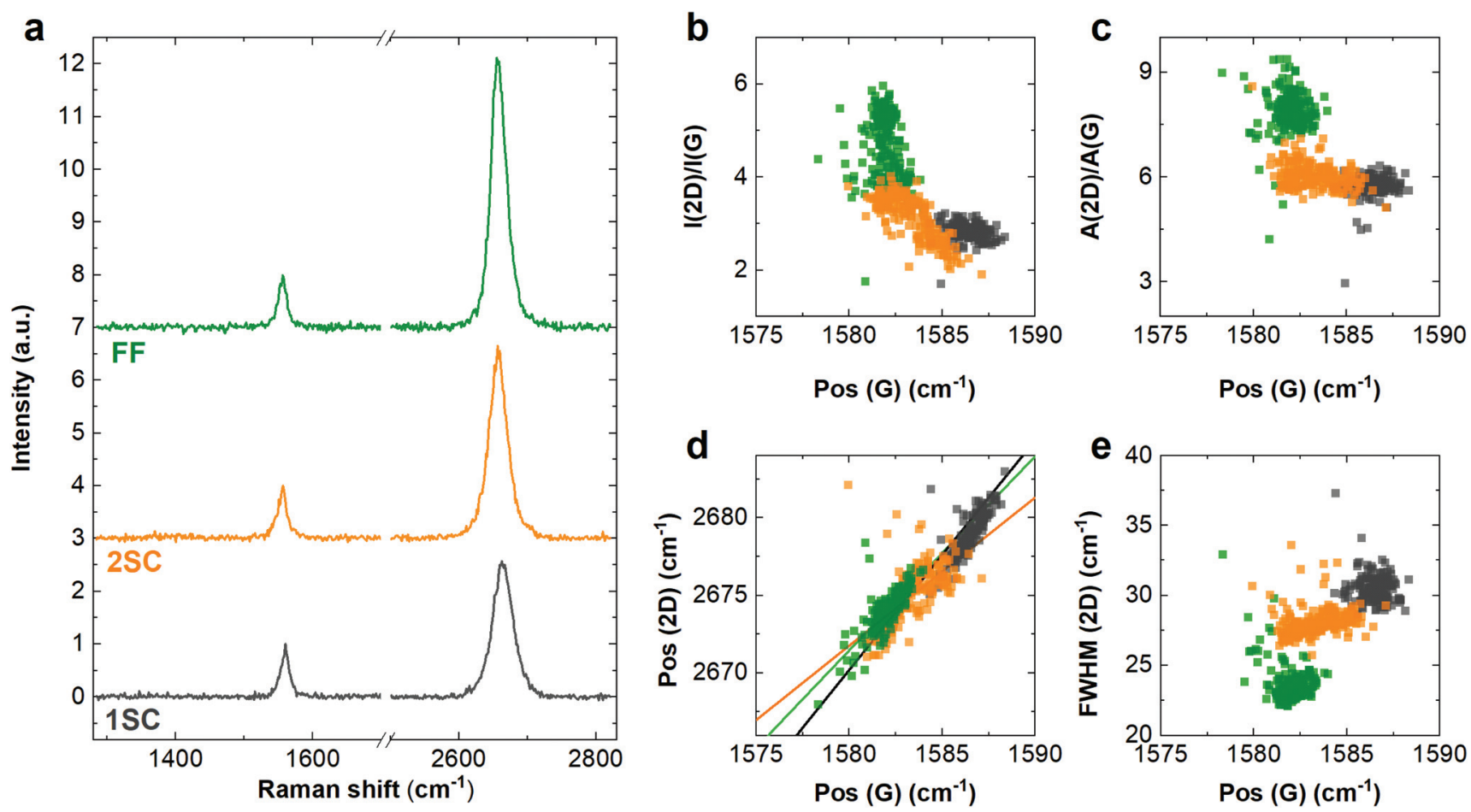

Fig. 2 (a) Raman spectra of graphene at various stages of processing: transfer and single-step cleaning, 1SC (black), two-step cleaning, 2SC (orange), full fabrication, FF (green). (b) 2D/G peak intensity ratio as a function of Pos(G) (c) 2D and G peak area ratio as a function of Pos(G) (d) Pos (2D) as a function of Pos(G). Solid lines show linear fits to the three data sets. (e) FWHM (2D) as a function of Pos(G).

(0\%) to $0.02 \%(0.01 \%)$ after 2 SC and $0 \%$ to $0.02 \%(0.01 \%)$ after full fabrication, respectively.

We also observe a significant reduction of average $2 \mathrm{D}$ width from FWHM(2D) $30.5 \pm 1.0 \mathrm{~cm}^{-1}$ for graphene after 1SC to FWHM(2D) $\sim 23.6 \pm 1.3 \mathrm{~cm}^{-1}$ for graphene after fabrication, as shown in Fig. 2c. FWHM(2D) is known to be sensitive to the strain variation within the area of the laser $\operatorname{spot}^{53}$ and is a good indication of the quality of on-substrate graphene layers. ${ }^{54}$ Notably, graphene on $\mathrm{SiO}_{2}$ typically shows higher FWHM(2D) $>25 \mathrm{~cm}^{-1}$, even for the case of exfoliated flakes. ${ }^{54,55}$ This indicates that our ultra-clean graphene presents remarkably low strain fluctuation, which is essential to achieve high carrier mobility. We note that no D peak can be seen after any of the steps, indicating that the process does not induce any measurable amount of defects. Comparison of Raman maps reveals that 2SC is effective at reducing doping in graphene as well as reducing strain inhomogeneity, consistent with the removal of polymeric residues observed in AFM. It should be noted that 2SC is effective not only after graphene transfer but after any of the fabrication steps where PMMA deposition is involved. PMMA deposition and removal with 1SC during any processing step leads to a Raman spectrum resembling that of graphene after transfer (see ESI $\dagger$ ), but treating the sample with 2SC always leads to reduced doping and strain inhomogeneity. Interestingly, performing several device fabrication steps using 2SC (i.e., performing several rounds of PMMA resist deposition and its complete removal) leads to an overall improvement of Raman characteristics (reduction of
FWHM(2D) and red-shift of Pos(G) towards values corresponding to pristine graphene $\left.{ }^{46}\right)$ compared to as-transferred graphene treated to 2SC. It should be noted that this effect is observed for the first 2 re-depositions of polymer, after which no improvement is observed. Similarly, treating graphene with AR 600-71 remover beyond the standard 3 minutes does not lead to further improvement. Additional Raman and AFM data taken during different processing steps can be found in ESI. $\dagger$

We also demonstrate that 2SC can be used to remove the PMMA residues from the surface of CVD grown polycrystalline graphene transferred to $\mathrm{SiO}_{2} / \mathrm{Si}$ using the standard wet etching approach, as shown in ESI. $\dagger$ Raman (Fig. S7†) and AFM (Fig. S6 $\dagger$ ) measurements indicate the reduction in doping and removal of PMMA residues, respectively. Finally, also in this case we observed that using separately the two constituents of remover AR 600-71 was less effective than using the commercial product, with 1,3-dioxolane yielding more sizable improvements in the Raman spectra.

\subsection{XPS analysis}

Surface chemical composition of graphene after 1SC and 2SC was investigated by XPS. In order to localize the domains, XPS Parallel Imaging $\left(500 \times 500 \mu \mathrm{m}^{2}\right)$ was used. From the $\mathrm{C} 1 \mathrm{~s}$ map recorded at $284.5 \mathrm{eV}$, a $60 \mu \mathrm{m}$ large area in the middle of a graphene flake was isolated and analysed by selected Small-area XPS (SAXPS). Fig. 3 shows the C 1s SAXPS spectrum (292-282 $\mathrm{eV}$ ) recorded on a graphene crystal for $1 \mathrm{SC}$ (blue) and 2SC (red) samples. Noting that the red continuous line with a broad 

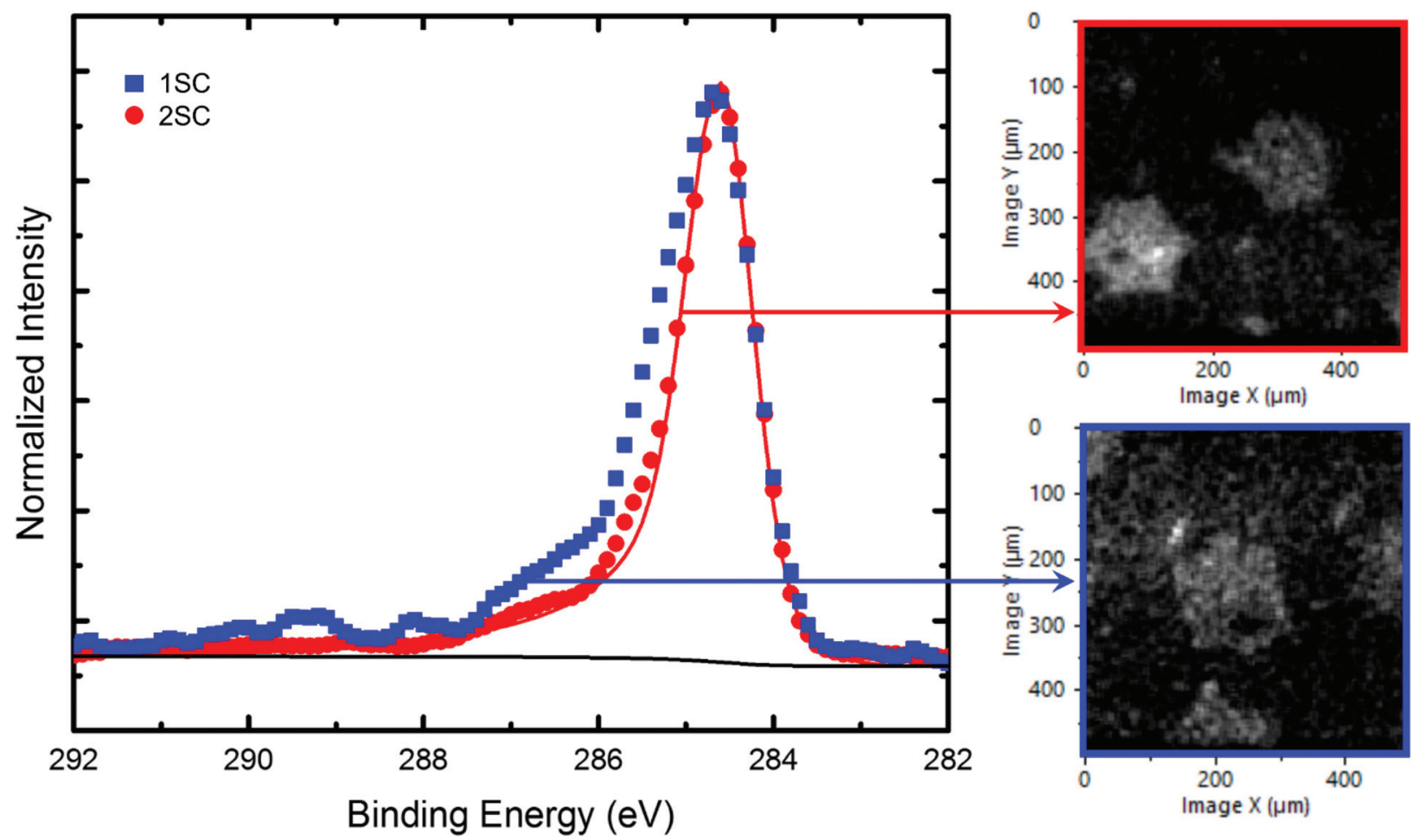

Fig. $3 \mathrm{C}$ 1s SAXPS spectra recorded on $60 \mu \mathrm{m}$ large area of graphene for samples after 1SC (blue) and 2SC (red). Positions were determined thanks to parallel XPS Imaging $\left(500 \times 500 \mathrm{um}^{2}\right)$ at $284.5 \mathrm{eV}$. In the spectra, the red and blue symbols represent the experimental data and the red straight line is related to the fit of a graphene component with an asymmetric shape.

asymmetric tail towards higher binding energy mimics a pure $\mathrm{sp}^{2}$-hybridized $\mathrm{C}-\mathrm{C}$ component (graphene), the C 1s spectra suggests that some components at higher energies related to PMMA residues are much more pronounced after 1 SC. ${ }^{42}$ Indeed, XPS analysis evidences a clear reduction of these spectral components after the second cleaning step, indicating its effectiveness in minimizing PMMA residues. The influence of the remover AR 600-71 is also visible on the C 1s XPS maps, which show better-defined flakes after 2SC.

In fact, on the $\mathrm{C}$ 1s image obtained after 1SC, PMMA residues remain on both the $\mathrm{SiO}_{2}$ substrate and on the graphene crystals, thus yielding a less-contrasted image. In order to ensure that contrasts are mainly related to chemical differences, Fig. S8 $\dagger$ shows C 1 s and Si $2 p$ XPS mapping on the same area. The opposite contrasts observed in the maps indicate that the observed differences are not governed by topographic effects.

\subsection{Electrical characterization}

To investigate the electrical properties of graphene, two sets of back-gated HBs were fabricated using electron beam lithography (EBL). One chip, shown in Fig. 4a, was processed using 2SC after transfer and each lithography step, whereas the reference chip was fabricated using $1 \mathrm{SC}$ at each step.

The field effect response of each $\mathrm{HB}$ with the channel aspect ratio $L / W=1$ was measured as a function of the backgate voltage using a 4-probe setup, by flowing a current $I_{\mathrm{SD}}=$
$1 \mu \mathrm{A}$ between the longitudinal contacts and measuring the voltage drop $V_{\mathrm{Xx}}$ along 2 adjacent side contacts. A schematic of the measurement configuration is shown in Fig. 4b. Resistance was calculated as $R=V_{\mathrm{XX}} / I_{\mathrm{SD}}$. Carrier mobility was calculated for each device as a function of carrier density $n$ (obtained from the applied back-gate bias) using the Drude formula:

$$
\mu=1 /(n e R),
$$

where $e$ is the electronic charge. Fig. 4c shows the field effect curve obtained for Hall Bar \#27 (HB27) on the 2SC chip, and Fig. $4 \mathrm{~d}$ shows the resulting carrier mobility as a function of $n$. Hole and electron mobility values at technologically-relevant carrier density of $1 \times 10^{12} \mathrm{~cm}^{-2}$ (ref. 55) are indicated on the curve. Residual charge density at CNP, $n^{*}$, was obtained for each device from a linear fit of conductivity on a double-logarithmic scale, as shown in Fig. 4e for HB27.

On average, the CNP was measured at $\sim 14.9 \pm 2.0 \mathrm{~V}$. This indicates p-type doping $\sim(1.0 \pm 0.1) \times 10^{12} \mathrm{~cm}^{-2}$, which is typical for high-quality non-encapsulated graphene measured in ambient conditions due to atmospheric adsorbates. ${ }^{31}$ Lower doping can be obtained by either encapsulating the graphene $^{56}$ or performing electrical characterisation in vacuum. ${ }^{31}$

Fig. 4f shows the measured hole and electron mobility values obtained at $n=1 \times 10^{12} \mathrm{~cm}^{-2}$ for all devices fabricated using 2SC (red and blue dots) and 1SC (black and orange dots). We plot the carrier mobility values as a function of calculated $n^{*}$, showing a clear inverse correlation between the 
a

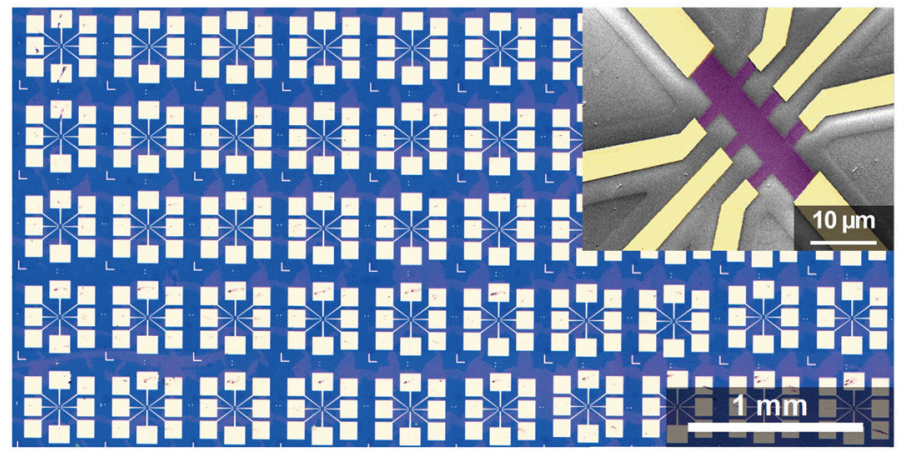

b

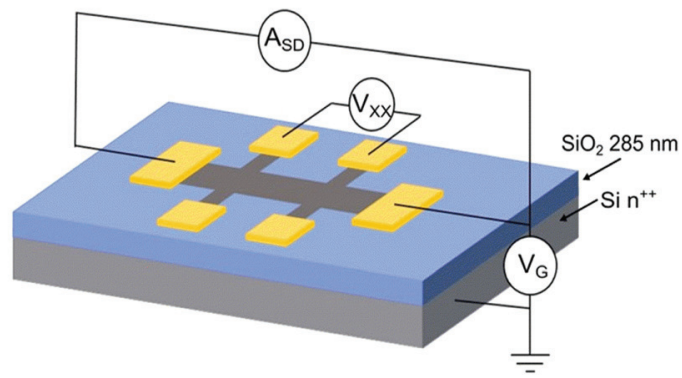

C

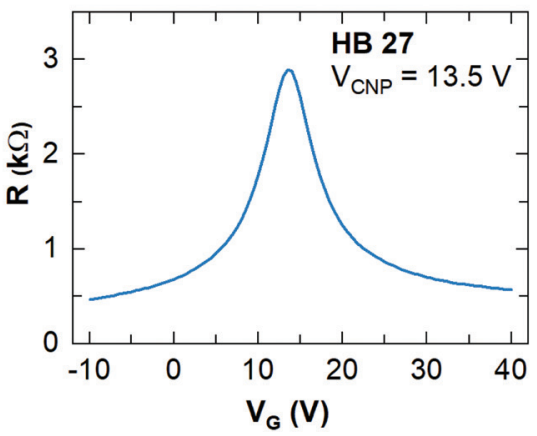

f

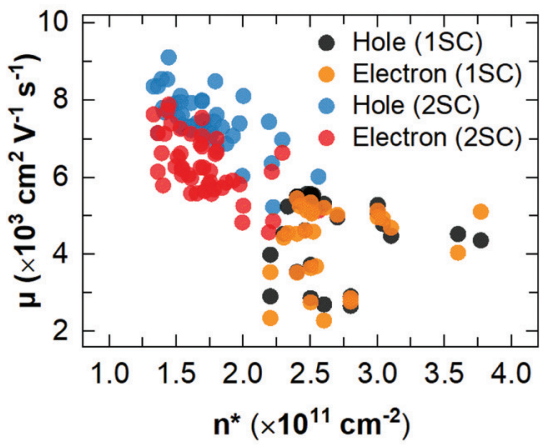

d

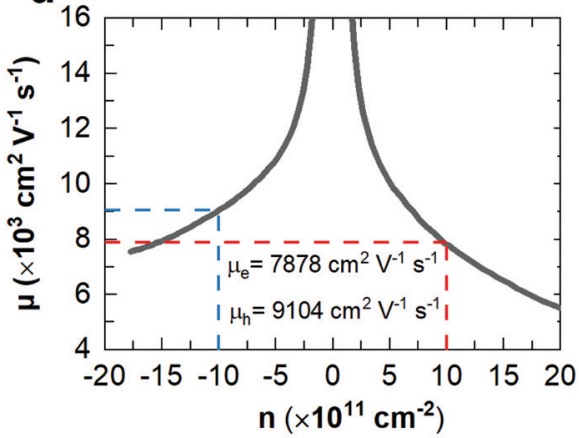

g

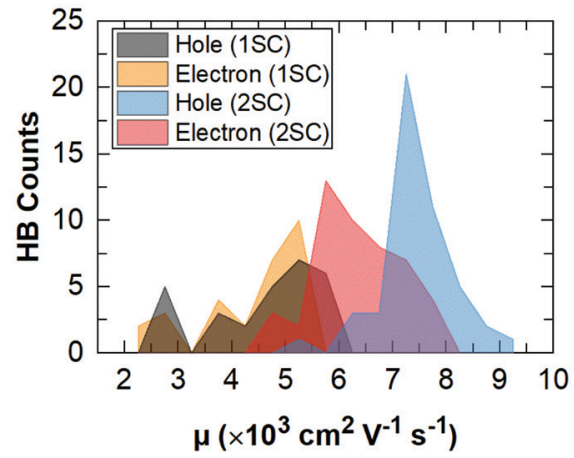

e

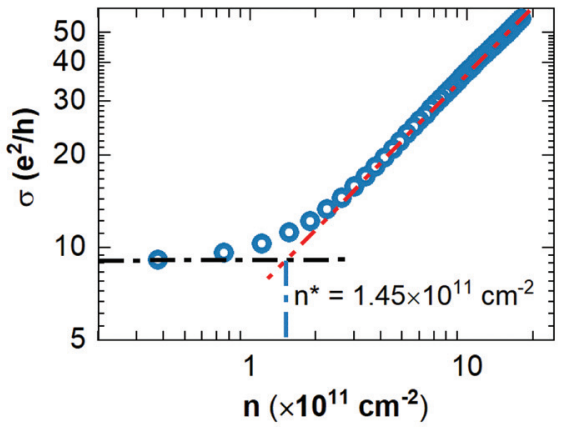

h

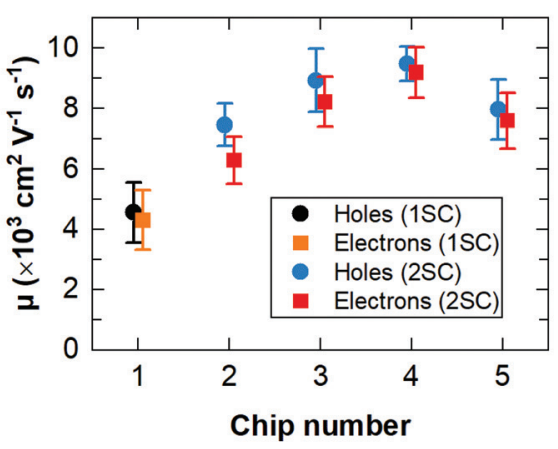

Fig. 4 Electrical characterization of devices fabricated using $1 \mathrm{SC}$ and $2 \mathrm{SC}$. (a) Optical image of 50 graphene $\mathrm{Hall}$ bars on $\mathrm{SiO} / 2 / \mathrm{Si}$. Inset: false-colour SEM image of a single Hall bar. (b) Schematic diagram of the 4-terminal electrical characterization setup. (c) Resistance curve of HB27 prepared with 2SC. (d) Carrier mobility as a function of carrier density calculated from the measurement in (c). (e) Linear fit of graphene conductivity as a function of carrier density to estimate the charge inhomogeneity $n^{*}$. (f) Mobility statistics of all graphene Hall bars prepared with 1SC (black, orange) and 2SC (red, blue) as a function of $n^{*}$. (g) Histogram of electron and hole mobility measured in chips fabricated using 1SC and 2SC. (h) Average electron and hole mobility measured in several samples processed using 1SC (chip 1) and 2SC (chips 2-5).

charge inhomogeneity and mobility, as observed in highquality graphene samples. ${ }^{53}$ For HBs fabricated using 2SC, average hole mobility $\mu_{\mathrm{h}}$ is estimated to be $\sim 7460 \pm 698 \mathrm{~cm}^{2}$ $\mathrm{V}^{-1} \mathrm{~s}^{-1}$, whereas electron mobility $\mu_{\mathrm{e}}$ is $\sim 6294 \pm 781 \mathrm{~cm}^{2} \mathrm{~V}^{-1}$ $\mathrm{s}^{-1}$ and average $n^{*} \sim(1.7 \pm 0.3) \times 10^{11} \mathrm{~cm}^{-2}$, with the best values reaching $\mu_{\mathrm{h}} \sim 9104 \mathrm{~cm}^{2} \mathrm{~V}^{-1} \mathrm{~s}^{-1}, \mu_{\mathrm{e}} \sim 7877 \mathrm{~cm}^{2} \mathrm{~V}^{-1} \mathrm{~s}^{-1}$ and $n^{*} \sim 1.4 \times 10^{11} \mathrm{~cm}^{-2}$. The high $\mu$ and low $n^{*}$ values are consistent with the low FWHM(2D) observed in the Raman measurements. Furthermore, these values compare favourably to the average values obtained from the sample fabricated using 1SC, namely $\mu_{\mathrm{h}} \sim 4562 \pm 1000 \mathrm{~cm}^{2} \mathrm{~V}^{-1} \mathrm{~s}^{-1}, \mu_{\mathrm{e}} \sim 4311 \pm$
$983 \mathrm{~cm}^{2} \mathrm{~V}^{-1} \mathrm{~s}^{-1}$ and $n^{*} \sim(2.9 \pm 0.4) \times 10^{11} \mathrm{~cm}^{-2}$. Fig. $4 \mathrm{~g}$ shows a histogram of electron and hole mobility measured in the sample processed with 1SC and the sample processed using 2SC. To further verify the advantage of using $2 \mathrm{SC}$ in graphene processing, we used 2SC to fabricate 3 other chips (in addition to the sample shown in Fig. 4a) with fewer test structures (5-8 Hall bars), all showing consistently high carrier mobility values $\left(\mu_{\mathrm{h}}>7500 \mathrm{~cm}^{2} \mathrm{~V}^{-1} \mathrm{~s}^{-1}, \mu_{\mathrm{e}}>6300 \mathrm{~cm}^{2} \mathrm{~V}^{-1} \mathrm{~s}^{-1}\right)$, as shown in Fig. $4 \mathrm{~h}$. The significantly improved mobility values of $2 \mathrm{SC}$ devices indicate that effective removal of polymer residues reduces charge-carrier scattering and unintentional 
doping. ${ }^{57,58}$ This demonstrates that $2 \mathrm{SC}$ enables graphene transfer and processing on a large scale with high mobility suitable for the fabrication of functional devices, such as optoelectronic modulators and photodetectors for high-speed telecommunications. ${ }^{9}$

\section{Conclusion}

In this work, we demonstrate an effective and rapid two-step cleaning (2SC) method to reduce the polymeric residues present on graphene surface after transfer and lithography processing. In this way, improved mobility can be achieved for CVD graphene on $\mathrm{SiO}_{2} / \mathrm{Si}$ substrates. AFM surface topography measurements and spatially-resolved XPS clearly show the effectiveness of this approach in removing PMMA residues, for both single-crystal graphene transferred with semi-dry transfer and polycrystalline graphene prepared with wet etching transfer. This makes the approach presented here a relevant technique for preparing high-quality graphene for various applications. A detailed analysis of Raman maps indicates the reduction of graphene doping after 2SC, while the absence of Raman D peak confirms that no structural defects are introduced in graphene. Electrical measurements show a significant improvement of the carrier mobility and residual charge carrier density with respect to chips processed using the traditional fabrication procedure. The 2SC approach does not introduce defects and yields high cleanliness while being scalable, rapid and easy to perform, with a great improvement over the existing approaches such as thermal annealing, scanning probe cleaning, and polymer-free fabrication (stencil mask lithography). Hence, it provides a straightforward route for the achievement of ultra-clean high-mobility scalable graphene devices which are sought after by several applications.

\section{Author contributions}

A. T. conceptualization, investigation, writing - original draft, visualization, V. M. conceptualization, investigation, writing reviewing and editing, visualization, supervision, L. M. investigation, writing - reviewing and editing, S. F. investigation, writing - reviewing and editing, N. M. investigation, writing - reviewing and editing, Z. M. G. investigation, writing - reviewing and editing, M. A. G. investigation, writing - reviewing and editing, J. Z. Investigation, writing - reviewing and editing, M. F. Investigation, writing reviewing and editing, D. A. supervision, writing - reviewing and editing, M. R. writing - reviewing and editing, supervision, F. B. writing - reviewing and editing, supervision, C. C. Conceptualization, writing - reviewing and editing, supervision.

\section{Conflicts of interest}

There are no conflicts to declare.

\section{Acknowledgements}

We acknowledge financial support from Fondazione Tronchetti Provera and National Centre for Scientific Research in the framework of the Emergence program at INC-CNRS. The research leading these results has received funding from the European Union Horizon 2020 Programme under grant agreement no. 881603 Graphene Core 3.

\section{References}

1 E. P. Randviir, D. A. C. Brownson and C. E. Banks, Mater. Today, 2014, 17, 426-432.

2 K. S. Novoselov, V. I. Fal'ko, L. Colombo, P. R. Gellert, M. G. Schwab and K. Kim, Nature, 2012, 490, 192-200.

3 D. M. A. Mackenzie, K. Smistrup, P. R. Whelan, B. Luo, A. Shivayogimath, T. Nielsen, D. H. Petersen, S. A. Messina and P. Bøggild, Appl. Phys. Lett., 2017, 111, 193103.

4 Y. Kim, T. Kim, J. Lee, Y. S. Choi, J. Moon, S. Y. Park, T. H. Lee, H. K. Park, S. A. Lee, M. S. Kwon, H. G. Byun, J. H. Lee, M. G. Lee, B. H. Hong and H. W. Jang, Adv. Mater., 2021, 33, 2004827.

5 S. Goossens, G. Navickaite, C. Monasterio, S. Gupta, J. J. Piqueras, R. Pérez, G. Burwell, I. Nikitskiy, T. Lasanta, T. Galán, E. Puma, A. Centeno, A. Pesquera, A. Zurutuza, G. Konstantatos and F. Koppens, Nat. Photonics, 2017, 11, 366-371.

6 A. Montanaro, W. Wei, D. De Fazio, U. Sassi, G. Soavi, P. Aversa, A. C. Ferrari, H. Happy, P. Legagneux and E. Pallecchi, Nat. Commun., 2021, 12, 2728.

7 V. Pistore, H. Nong, P. B. Vigneron, K. Garrasi, S. Houver, L. Li, A. G. Davies, E. H. Linfield, J. Tignon, J. Mangeney, R. Colombelli, M. S. Vitiello and S. S. Dhillon, Nat. Commun., 2021, 12, 1427.

8 M. A. Giambra, V. Mišeikis, S. Pezzini, S. Marconi, A. Montanaro, F. Fabbri, V. Sorianello, A. C. Ferrari, C. Coletti and M. Romagnoli, ACS Nano, 2021, 15, 31713187.

9 M. Romagnoli, V. Sorianello, M. Midrio, F. H. L. Koppens, C. Huyghebaert, D. Neumaier, P. Galli, W. Templ, A. D'Errico and A. C. Ferrari, Nat. Rev. Mater., 2018, 3, 392414.

10 V. Mišeikis, S. Marconi, M. A. Giambra, A. Montanaro, L. Martini, F. Fabbri, S. Pezzini, G. Piccinini, S. Forti, B. Terrés, I. Goykhman, L. Hamidouche, P. Legagneux, V. Sorianello, A. C. Ferrari, F. H. L. Koppens, M. Romagnoli and C. Coletti, ACS Nano, 2020, 14, 11190-11204.

11 M. A. Giambra, V. Sorianello, V. Miseikis, S. Marconi, A. Montanaro, P. Galli, S. Pezzini, C. Coletti and M. Romagnoli, Opt. Express, 2019, 27, 20145.

12 G. Lupina, J. Kitzmann, I. Costina, M. Lukosius, C. Wenger, A. Wolff, S. Vaziri, M. Östling, I. Pasternak, A. Krajewska, W. Strupinski, S. Kataria, A. Gahoi, M. C. Lemme, G. Ruhl, G. Zoth, O. Luxenhofer and W. Mehr, ACS Nano, 2015, 9, 4776-4785. 
13 N. Mishra, S. Forti, F. Fabbri, L. Martini, C. McAleese, B. R. Conran, P. R. Whelan, A. Shivayogimath, B. S. Jessen, L. Buß, J. Falta, I. Aliaj, S. Roddaro, J. I. Flege, P. Bøggild, K. B. K. Teo and C. Coletti, Small, 2019, 15, 1970273.

14 B. H. Son, H. S. Kim, H. Jeong, J. Y. Park, S. Lee and Y. H. Ahn, Sci. Rep., 2017, 7, 18058.

15 Y.-C. C. Lin, C.-C. C. Lu, C.-H. H. Yeh, C. Jin, K. Suenaga and P.-W. W. Chiu, Nano Lett., 2012, 12, 414-419.

16 A. Pirkle, J. Chan, A. Venugopal, D. Hinojos, C. W. Magnuson, S. McDonnell, L. Colombo, E. M. Vogel, R. S. Ruoff and R. M. Wallace, Appl. Phys. Lett., 2011, 99, 122108.

17 L. Gammelgaard, J. M. Caridad, A. Cagliani, D. M. A. MacKenzie, D. H. Petersen, T. J. Booth and P. Bøggild, 2D Mater., 2014, 1, 035005.

18 W. Choi, M. A. Shehzad, S. Park and Y. Seo, RSC Adv., 2017, 7, 6943-6949.

19 A. M. Goossens, V. E. Calado, A. Barreiro, K. Watanabe, T. Taniguchi and L. M. K. Vandersypen, Appl. Phys. Lett., 2012, 100, 073110.

20 J. Moser, A. Barreiro and A. Bachtold, Appl. Phys. Lett., 2007, 91, 163513.

21 B. Zhuang, S. Li, S. Li and J. Yin, Carbon, 2021, 173, 609636.

22 X. Yang and M. Yan, Nano Res., 2020, 13, 599-610.

23 Z. Cheng, Q. Zhou, C. Wang, Q. Li, C. Wang and Y. Fang, Nano Lett., 2011, 11, 767-771.

24 K. Kumar, Y. S. Kim and E. H. Yang, Carbon, 2013, 65, 3545.

25 W. Choi, Y. S. Seo, J. Y. Park, K. B. Kim, J. Jung, N. Lee, Y. Seo and S. Hong, IEEE Trans. Nanotechnol., 2015, 14, 7074.

26 J. W. Suk, W. H. Lee, J. Lee, H. Chou, R. D. Piner, Y. Hao, D. Akinwande and R. S. Ruoff, Nano Lett., 2013, 13, 14621467.

27 M. Her, R. Beams and L. Novotny, Phys. Lett. A, 2013, 377, 1455-1458.

28 Z. H. Ni, H. M. Wang, Z. Q. Luo, Y. Y. Wang, T. Yu, Y. H. Wu and Z. X. Shen, J. Raman Spectrosc., 2010, 41, 479483.

29 H. Park, C. Lim, C.-J. Lee, J. Kang, J. Kim, M. Choi and H. Park, Nanotechnology, 2018, 29, 415303.

30 L. Lin, J. Zhang, H. Su, J. Li, L. Sun, Z. Wang, F. Xu, C. Liu, S. Lopatin, Y. Zhu, K. Jia, S. Chen, D. Rui, J. Sun, R. Xue, P. Gao, N. Kang, Y. Han, H. Q. Xu, Y. Cao, K. S. Novoselov, Z. Tian, B. Ren, H. Peng and Z. Liu, Nat. Commun., 2019, 10, 1912.

31 B. Chen, H. Huang, X. Ma, L. Huang, Z. Zhang and L.-M. Peng, Nanoscale, 2014, 6, 15255-15261.

32 T. Wu, G. Ding, H. Shen, H. Wang, L. Sun, D. Jiang, X. Xie and M. Jiang, Adv. Funct. Mater., 2013, 23, 198203.

33 E. N. D. de Araujo, T. A. S. L. de Sousa, L. D. M. Guimarães and F. Plentz, Beilstein J. Nanotechnol., 2019, 10, 349-355.

34 W. Wu, L. A. A. Jauregui, Z. Su, Z. Liu, J. Bao, Y. P. P. Chen and Q. Yu, Adv. Mater., 2011, 23, 4898-4903.
35 Q. Yu, L. a Jauregui, W. Wu, R. Colby, J. Tian, Z. Su, H. Cao, Z. Liu, D. Pandey, D. Wei, T. F. Chung, P. Peng, N. P. Guisinger, E. a Stach, J. Bao, S.-S. Pei and Y. P. Chen, Nat. Mater., 2011, 10, 443-449.

36 V. Miseikis, D. Convertino, N. Mishra, M. Gemmi, T. Mashoff, S. Heun, N. Haghighian, F. Bisio, M. Canepa, V. Piazza and C. Coletti, 2D Mater., 2015, 2, 014006.

37 V. Miseikis, F. Bianco, J. David, M. Gemmi, V. Pellegrini, M. Romagnoli and C. Coletti, 2D Mater., 2017, 4, 021004.

38 Y. Wang, Y. Zheng, X. Xu, E. Dubuisson, Q. Bao, J. Lu and K. P. Loh, ACS Nano, 2011, 5, 9927-9933.

39 L. Gao, W. Ren, H. Xu, L. Jin, Z. Wang, T. Ma, L.-P. Ma, Z. Zhang, Q. Fu, L.-M. Peng, X. Bao and H.-M. Cheng, Nat. Commun., 2012, 3, 699.

40 Information sheet - Remover for AR Resists, https://www. allresist.com/wp-content/uploads/sites/2/2016/12/homepage_allresist_produktinfos_csar_electra_7520_englisch.pdf, (accessed March 2021).

41 C. H. Lui, L. Liu, K. F. Mak, G. W. Flynn and T. F. Heinz, Nature, 2009, 462, 339-341.

42 G. Cunge, D. Ferrah, C. Petit-Etienne, A. Davydova, H. Okuno, D. Kalita, V. Bouchiat and O. Renault, J. Appl. Phys., 2015, 118, 123302.

43 A. C. Ferrari, J. C. Meyer, V. Scardaci, C. Casiraghi, M. Lazzeri, F. Mauri, S. Piscanec, D. Jiang, K. S. Novoselov, S. Roth and A. K. Geim, Phys. Rev. Lett., 2006, 97, 187401.

44 L. G. Cançado, A. Jorio, E. H. M. Ferreira, F. Stavale, C. A. Achete, R. B. Capaz, M. V. O. Moutinho, A. Lombardo, T. S. Kulmala and A. C. Ferrari, Nano Lett., 2011, 11, 31903196.

45 D. M. Basko, S. Piscanec and A. C. Ferrari, Phys. Rev. B: Condens. Matter Mater. Phys., 2009, 80, 165413.

46 A. Das, S. Pisana, B. Chakraborty, S. Piscanec, S. K. Saha, U. V. Waghmare, K. S. Novoselov, H. R. Krishnamurthy, A. K. Geim, A. C. Ferrari and A. K. Sood, Nat. Nanotechnol., 2008, 3, 210-215.

47 T. M. G. Mohiuddin, A. Lombardo, R. R. Nair, A. Bonetti, G. Savini, R. Jalil, N. Bonini, D. M. Basko, C. Galiotis, N. Marzari, K. S. Novoselov, A. K. Geim and A. C. Ferrari, Phys. Rev. B: Condens. Matter Mater. Phys., 2009, 79, 205433.

48 J. Zabel, R. R. Nair, A. Ott, T. Georgiou, A. K. Geim, K. S. Novoselov and C. Casiraghi, Nano Lett., 2012, 12, 617621.

49 D. Yoon, Y. W. Son and H. Cheong, Phys. Rev. Lett., 2011, 106, 155502.

50 J. E. Lee, G. Ahn, J. Shim, Y. S. Lee and S. Ryu, Nat. Commun., 2012, 3, 1024.

51 A. C. Ferrari and D. M. Basko, Nat. Nanotechnol., 2013, 8, 235-246.

52 S. Piscanec, M. Lazzeri, F. Mauri, A. C. Ferrari and J. Robertson, Phys. Rev. Lett., 2004, 93, 185503.

53 C. Neumann, S. Reichardt, P. Venezuela, M. Drögeler, L. Banszerus, M. Schmitz, K. Watanabe, T. Taniguchi, F. Mauri, B. Beschoten, S. V. Rotkin and C. Stampfer, Nat. Commun., 2015, 6, 8429. 
54 N. J. G. Couto, D. Costanzo, S. Engels, D. K. Ki, K. Watanabe, T. Taniguchi, C. Stampfer, F. Guinea and A. F. Morpurgo, Phys. Rev. X, 2014, 4, 041019.

55 J. Chen, C. Jang, S. Xiao, M. Ishigami and M. S. Fuhrer, Nat. Nanotechnol., 2008, 3, 206-209.

56 A. A. Sagade, D. Neumaier, D. Schall, M. Otto, A. Pesquera, A. Centeno, A. Z. Elorza and H. Kurz, Nanoscale, 2015, 7, 3558-3564.
57 H. Sojoudi, J. Baltazar, C. Henderson and S. Graham, J. Vac. Sci. Technol., B: Nanotechnol. Microelectron.: Mater., Process., Meas., Phenom., 2012, 30, 041213.

58 S. Ryu, L. Liu, S. Berciaud, Y. J. Yu, H. Liu, P. Kim, G. W. Flynn and L. E. Brus, Nano Lett., 2010, 10, 49444951.

59 V. Mišeikis and C. Coletti, Appl. Phys. Lett., 2021, 119, 050501 . 